\title{
Kirenol Inhibits the Function and Inflammation of Fibroblast-like Synoviocytes in Rheumatoid Arthritis in vitro and in vivo
}

\author{
Jing $\mathrm{Wu}^{1+}$, Qiang $\mathrm{Li}^{1+}$, Li Jin ${ }^{1}$, Yuan $\mathrm{Qu}{ }^{1}$, Bi-Bo Liang ${ }^{1}$, Xiao-Tong Zhu ${ }^{1}$, Hong-Yan Du ${ }^{2}$, \\ Li-Gang Jie ${ }^{1 *}$ and Qing-Hong Yu ${ }^{1 *}$
}

${ }^{1}$ Rheumatology and Clinical Immunology, ZhuJiang Hospital, Southern Medical University, Guangzhou, China, ${ }^{2}$ School of Laboratory Medicine and Biotechnology, Southern Medical University, Guangzhou, China

OPEN ACCESS

Edited by:

Hanshi Xu,

First Affiliated Hospital of Sun Yat-sen

University, China

Reviewed by:

Wenfeng Tan

Nanjing Medical University, China

Balik Dzhambazov,

Plovdiv University Paisii Hilendarski,

Bulgaria

*Correspondence:

Li-Gang Jie

jieligang@hotmail.com

Qing-Hong Yu

yuqinghong@smu.edu.cn

tThese authors have contributed equally to this work

Specialty section:

This article was submitted to Autoimmune and Autoinflammatory

Disorders,

a section of the journal

Frontiers in Immunology

Received: 04 March 2019 Accepted: 22 May 2019

Published: 06 June 2019

Citation:

Wu J, Li Q, Jin L, Qu Y, Liang B-B, Zhu X-T, Du H-Y, Jie L-G and Yu Q-H (2019) Kirenol Inhibits the Function and Inflammation of Fibroblast-like Synoviocytes in Rheumatoid Arthritis

in vitro and in vivo.

Front. Immunol. 10:1304.

doi: 10.3389/fimmu.2019.01304
Kirenol is a diterpenoid extracted from the Chinese herbal medicine Siegesbeckiae. Siegesbeckiae has been used to treat Rheumatoid arthritis (RA) in China for several centuries. RA is characterized by the proliferation of synoviocytes in inflamed synovia, as well as by their expression of inflammatory cytokines. In the present study, we found that Kirenol inhibited the migration, invasion, and proinflammatory of IL-6 secretion of RA-associated synovial fibroblasts (FLS) at a concentration of $100-200 \mu \mathrm{g} / \mathrm{ml}$ in vitro. Proinflammatory cytokines production and synovium hyperplasia and cartilage erosion were also inhibited in a collagen-induced arthritis (CIA) mouse model upon Kirenol treatment. Together, our results thus confirm that Kirenol has potent therapeutic efficacy in RA owing to its ability to suppress negative FLS activities.

Keywords: kirenol, Rheumatoid arthritis, fibroblast-like synoviocytes, IL-6, migration, invasion

\section{INTRODUCTION}

RA is a chronic and refractory autoimmune joint disease characterized by the proliferation of synoviocytes in the inflamed synovia, and by the expression of inflammatory cytokines on these cells (1). Synovial fibroblasts (FLS) promote joint destruction via their attachment to the cartilage, and thus are key mediators of the pathogenesis of RA (2). Although there are many RA treatment options available, including traditional disease-modifying antirheumatic drugs (DMARDs) as well new and effective biologicals agents, these treatments ultimately induce remission in only $20-68 \%$ of patients (3). Moreover, there is still ample opportunity for the development of novel drugs capable of inhibiting synovial hyperplasia. Kirenol is a diterpenoid compound derived from Herba Siegesbeckia that has been traditionally used in China to treat RA for centuries. Kirenol has been suggested to exhibit primary anti-inflammatory and anti-rheumatic activities $(4,5)$. The active ingredient Kirenol in Herba Siegesbeckia extracts was shown to reduce the inflammatory pathology in collagen induced arthritis (CIA) model rats, and additional studies suggest that Kirenol is able to suppress the production of IL- $1 \beta$ and TNF- $\alpha$ in the serum of adjuvant arthritis model animals $(5,6)$. While multiple studies have thus demonstrated the anti-inflammatory properties of Kirenol, there is still very limited information available regarding the specific mechanisms and dynamics whereby Kirenol affects RA-associated FLS cells both in vitro and in vivo. The inflammatory milieu in the synovial compartment is regulated by a complex cytokine network. Many pro-inflammatory cytokines such as TNF- $\alpha$, IL- $1 \beta$, and IL- 6 , are thought to contribute to the pathological 
development and progression of RA (7). Activated FLS cells secrete large quantities of IL- 6 and IL- 8 (8). Antibodies directed against TNF- $\alpha$ and IL- 6 have shown efficacy for the treatment of $\mathrm{RA}$, consistent with the fact that joint destruction is positively correlated with pro-inflammatory cytokine levels in the serum or synovial tissue. Multiple cytokines and matrix metalloproteinases (MMPs) are present in the synovium of RA patients, where they play important roles in the maintenance of inflammatory responses $(9,10)$. Certain proteins and cytokines, including IL6 , IL- 8 , TNF- $\alpha$, IL-1 $\beta$, and MMP-1, 2, 3, 9 have been identified as diagnostic indicators of RA and as possible therapeutic targets. As such, any effort to determine how Kirenol affects FLS cells necessitates an investigation of its effects on cytokine production.

The pathophysiology of RA involves chronic inflammation and pannus formation in the synovial membrane, which can lead to the destruction of articular cartilage and bone. As these proinflammatory cytokines and MMPs are specifically involved in the pathogenesis in RA and are highly expressed in the serum and synovial fluid of RA patients, we hypothesized that these factors may be downregulated by Kirenol. The aims of this study were therefore to evaluate whether Kirenol treatment leads to decreased production of these factors by RA-associated FLS cells, and to explore the underlying molecular mechanisms governing such regulatory activity.

\section{MATERIALS AND METHODS}

\section{Human FLS Culture}

Synovial tissue samples were obtained from the knees of five patients with active RA (as diagnosed according to the 2010 Rheumatoid arthritis classification criteria) during knee joint arthroscopic operations. The synovial tissue was cut into 1-2 $\mathrm{mm}^{3}$ pieces and distributed evenly in a culture flask. After $4 \mathrm{~h}$, this flask was inverted and the synovial tissue was cultured in DMEM containing $10 \%$ fetal calf serum, $100 \mathrm{U} / \mathrm{ml}$ penicillin, and $100 \mu \mathrm{g} / \mathrm{ml}$ streptomycin in a humid incubator containing $5 \% \mathrm{CO}_{2}$. Cell media was changed every 3-4 days. The FLS cells were grown in a monolayer, and cells between the third and sixth generations were used for all experiments.

\section{Cell Viability Assays}

Cell viability was detected using the CCK-8 kit (Dojindo, China) according to the provided instructions. Briefly, cultured RA-FLS cells were plated in 96-well plates at a density of $1 \times 10^{3}$ cells/well in DMEM containing $10 \%$ FBS. Cells were then incubated with Kirenol (50, 100, and $200 \mu \mathrm{g} / \mathrm{ml}$; Herbpurity, China) for another $24 \mathrm{~h}$. Human IL-17A (100 ng/ml, R\&D, USA) and TNF$\alpha(100 \mathrm{ng} / \mathrm{ml}, \mathrm{R} \& \mathrm{D}, \mathrm{USA})$ were used as positive controls. After this incubation period, $10 \mu \mathrm{L}$ of the CCK- 8 solution was added to each well and cells were incubated for $4 \mathrm{~h}$. The absorbance at $450 \mathrm{~nm}$ was then measured via a microplate reader.

\section{Quantitative PCR}

RA-FLS cells were seeded in 24-well plates at a density of $2 \times$ $10^{4}$ cells/well for $24 \mathrm{~h}$, and were then treated with Kirenol at concentrations of 50,100 , or $200 \mu \mathrm{g} / \mathrm{ml}$ for $4 \mathrm{~h}$, with positive controls employed as above. Total FLS RNA was then isolated at appropriates using the Trizol reagent (Invitrogen, USA) according to the manufacturer's protocols. Reverse transcription was conducted using a first-strand cDNA synthesis kit (TaKaRa, China). To assess IL-6, IL-8, MMP1, MMP2, MMP3, MMP9, NFкB P50, NFкB P65, MAPK, JNK, and JAK expression, realtime PCR was performed using a SYBR Premix ExTaq kit (TaKaRa, China). Resultant heatmap figures were prepared using the R software [package(heatmap)].

\section{ELISAs}

After being treated as described above, $2 \times 10^{4}$ RA-FLS cells were treated with Kirenol for 4 or $24 \mathrm{~h}$. Supernatants were then collected to measure IL-6, IL- 8 , IL- $1 \beta$, and TNF- $\alpha$. For in vivo experiments, murine serum was similarly used for cytokine detection. ELISA kits used included those specific for IL-1 $\beta$ (R\&D, USA), TNF- $\alpha$ (Thermo Fisher Scientific, USA), IL-6 (Thermo Fisher Scientific, USA), IL-8 (Thermo Fisher Scientific, USA). The optical density (OD) value for each sample was determined at $450 \mathrm{~nm}$.

\section{Western Blotting}

RIPA lysis buffer (50 mM Tris-Cl pH 7.4, $150 \mathrm{mM} \mathrm{NaCl}$, $1 \%$ Triton $\mathrm{X}-100,1 \%$ sodium deoxycholate, $0.1 \%$ SDS), containing protease and phosphatase inhibitors as well as phenylmethanesulfonyl fluoride (PMSF), was used to lyse and collect protein from cell samples. Protein was then loaded onto $8 \%$ polyacrylamide Tris/glycine gels and separated at $80 \mathrm{~V}$ for $30 \mathrm{~min}$, followed by $110 \mathrm{~V}$ for $1 \mathrm{~h}$, and samples were then transferred to a nitrocellulose membrane at $100 \mathrm{~V}$ for $2 \mathrm{~h}$. After blocking, the membranes were probed using the MAPK Family Antibody Sampler Kit (Cell Signaling Technology, USA), NF-кB Pathway Sampler Kit (Cell Signaling Technology, USA), or Phospho-Jak Family Antibody Sampler Kit (Cell Signaling Technology, USA). Phospho-antibodies were diluted to 1:100, while all others were diluted to 1:500. After chemiluminescence development (SignalFire ECL Reagent, Cell Signaling Technology, USA), gel images were scanned and analyzed using the Image J (v1.52) image processing software.

Murine synovial tissues were taken from around the hip joints, as described in our previously research method (11). For murine synovium samples, western blotting was performed as above, using IL-6, IL-8, and TNF- $\alpha$ antibodies purchased from Biomathematics and Statistics Scotland (China).

\section{Measures of FLS Migration and Invasion Wound Healing Assay}

To demonstrate the effects of Kirenol on the migratory capacity of FLS, a wound healing assay was performed. $2 \times 10^{5}$ /well RAFLS were seeded in 24-well plates for $24 \mathrm{~h}$, after which a $200 \mu \mathrm{l}$ pipette tip was used to create a straight scratch wound in the monolayer. Cells were then incubated with Kirenol (50, 100, or $200 \mu \mathrm{g} / \mathrm{ml}$ ) for an additional $48 \mathrm{~h}$, with cell being imaged after 0 , 4,24 , and $48 \mathrm{~h}$. Image J (v1.52) was used to analyze the migratory wound healing dynamics. 


\section{Transwell Migration and Invasion Assays}

To further explore the effects of Kirenol on cellular responses, chemotaxis assays were performed using transwell chambers with an $8.0 \mu \mathrm{m}$ pore size (Corning, USA). Cells were incubated with Kirenol concentrations as described above for $24 \mathrm{~h}$, and then a total of $2 \times 10^{5}$ FLS in serum-free DMEM were added to the upper chambers of these Transwell systems for $8 \mathrm{~h}$. In addition, $600 \mu \mathrm{l}$ of DMEM medium containing $10 \%$ FBS was added to the lower chamber of each well in a 24-well plate. In parallel, similar invasion assays were performed using an $8.0 \mu \mathrm{m}$ PET membrane (Corning, USA). For this experiment, FLS were seeded at a density of $1 \times 10^{5} / \mathrm{ml}$ and were grown in DMEM for $12 \mathrm{~h}$. Cells that failed to migrate were removed with a cotton swab, after which the membranes were fixed with $4 \%$ paraformaldehyde for $30 \mathrm{~min}$ and then stained with $0.1 \%$ crystal violet. Migration was quantified by counting the number of stained cells that had migrated to the lower side of the filter using an optical microscope. The average of the number of invading cells from the six random fields of view after normalization to control were used to determine rates of chemotaxis/invasion.

\section{Murine Experiments}

\section{Arthritis Model Development}

CIA was induced in 9-week-old male DBA/1 mice. Mice were purchased from HuFukang Biotechnology Co., China (license number: SCXK (Jing) 2014-0004). All experiments were performed in accordance with the guidelines of the local animal ethics committee. A total of 15 mice were divided into 3 groups, and received $0,7.5$, or $30 \mathrm{mg} / \mathrm{kg}$ Kirenol q.d. Mice were treated with Kirenol for 1 week before being immunized with $100 \mu \mathrm{g}$ of bovine type II collagen and complete Freund's adjuvant (CFA; 1:1, Xinbosheng, China and Sigma, Japan) by injection at the tail base. A booster injection was administered on day 21, at which time a total of $100 \mu \mathrm{g}$ collagen II was administered in Freund's incomplete adjuvant (Sigma, Japan). Assessment of arthritis in each limb of these arthritic model mice was then performed via visual scoring from 0 to 4 . A maximal score for an individual animal was 16 (12). The weight of each mouse was also recorded once per week.

\section{Histological Scoring}

Joints were removed from CIA model mice and fixed in $10 \%$ formalin, after which they were decalcified in 10\% EDTA, embedded in paraffin, and stained with hematoxylin and eosin (H\&E) for light microscopy. Infiltration of inflammatory cells, transformation of the synovial lining, cartilage destruction, and pannus formation were independently scored in a blinded manner from 0 to 3 as in previous studies (13). Of the four limbs analyzed per animal, the maximum score for each category was used, with a maximum possible histological score of 12. Synovial inflammation and bone erosion scores were also performed as described previously, with a maximum possible score of 4 (14). Synovial inflammation was scored as follows: 0- no inflammation; 1- slight synovitis with some cell infiltration; 2 - moderate synovitis with moderate cell infiltration; 3- extensive synovitis with a moderate number of infiltrating cells; 4- extensive and severe synovitis, with the presence of numerous inflammatory cells. Bone erosion was scored as follows: 0- no erosion; 1 - small areas of resorption; 2- numerous areas of resorption; 3- extensive osteolysis; 4- extensive and severe osteolysis.

\section{Immunohistochemistry}

Joints sections were deparaffinized and washed with Trisbuffered saline (TBS) for $10 \mathrm{~min}$ and distilled water for $10 \mathrm{~min}$, after which antigen retrieval was performed via heating samples in citrate buffer for $15 \mathrm{~min}$. Samples were then incubated with primary antibodies against IL-6 (Servicebio, USA), IL-8 (Servicebio, USA), and TNF- $\alpha$ (Servicebio, USA), (1:50) at $4^{\circ} \mathrm{C}$ for $12 \mathrm{~h}$, followed by incubation with a secondary antibody (goat anti rabbit, 1:50, servicebio, USA) at room temperature for $50 \mathrm{~min}$. For antigen visualization, DAB solution was used for color development for $5 \mathrm{~min}$, after which the Image J (v1.52) and IHC Toolbox.jar (USA) programs were used for image analysis.

\section{Statistical Analysis}

Data are presented as means \pm standard deviation. Differences among groups were analyzed via the Kruskal-Wallis test (more than two groups) or Mann-Whitney $U$-test (two groups) using GraphPad Prism v5.0 (USA). Differences were considered to be statistically significant at $p<0.05$.

\section{RESULTS}

\section{Kirenol Inhibits RA-FLS Proliferation}

To determine whether Kirenol affects the proliferation of FLS, cells were stimulated with Kirenol $(50,100$, or $200 \mu \mathrm{g} / \mathrm{ml})$ and control in medium containing DMEM. As shown in Figure 1A, Kirenol impaired the proliferation of FLS in a dose-dependent manner. Even when FLS were stimulated with inflammatory cytokines as a positive control (TNF- $\alpha 100 \mathrm{ng} / \mathrm{ml}$ and IL17A $100 \mathrm{ng} / \mathrm{ml}$ ), Kirenol was still able to mildly inhibit their proliferation (Figures 1B,C).

\section{Kirenol Inhibits the Secretion of Cytokines by RA-FLS}

As shown in Figures 1D-F, Kirenol inhibited the secretion of IL- 6 by FLS with a dose-dependent manner even when cells were stimulated using TNF- $\alpha$ and IL-17A. However, Kirenol only significantly inhibited FLS IL-8 secretion after $30 \mathrm{~min}$ (Figure 1G), and no changes in IL- 8 secretion were observed at any time in the TNF- $\alpha$ and IL-17A-stimulated groups. We were not able to detect significant levels of IL- $1 \beta$ or TNF- $\alpha$ in FLS supernatants at any time.

\section{Kirenol Downregulates IL-6, IL-8, MMP-9, MAPK, P65, P50, and JAK Expression in RA-FLS}

RT-PCR was performed to assess the expression of IL-6, IL8, MMP-1, 2, 3, 9, NFкBP65, P50, MAPK, and JAK in FLS cells treated with Kirenol. We found that expression of IL6 and IL- 8 were down-regulated by Kirenol in the presence or absence of IL-17A and TNF- $\alpha$ stimulation (Figure 2A). Similarly, MMP-9, NFאB, MAPK, and JAK were down-regulated 

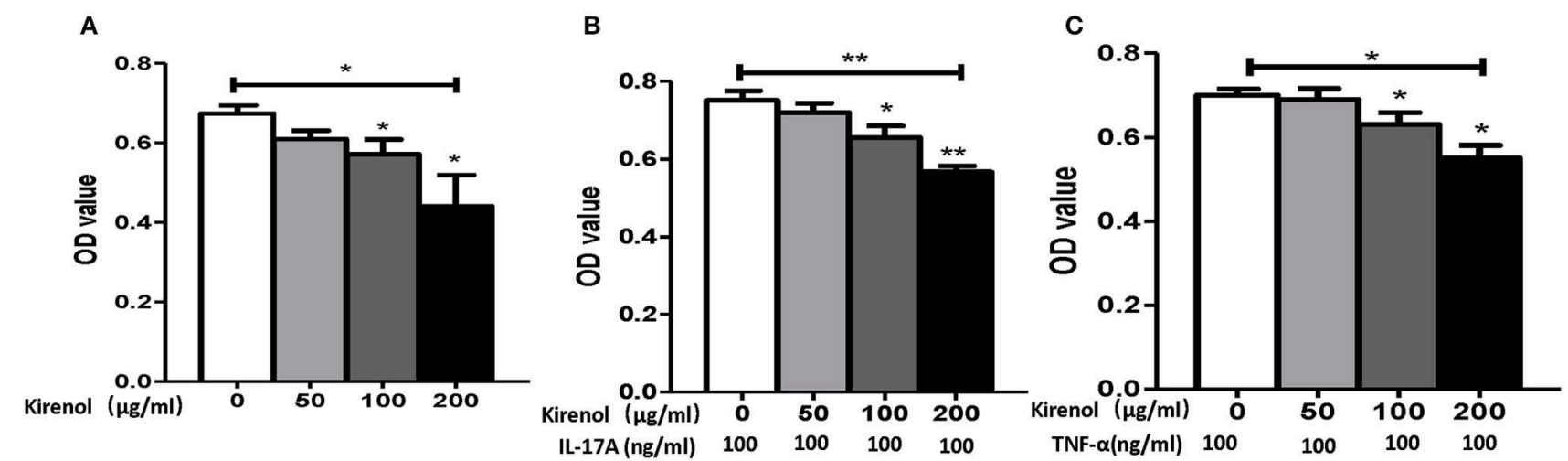

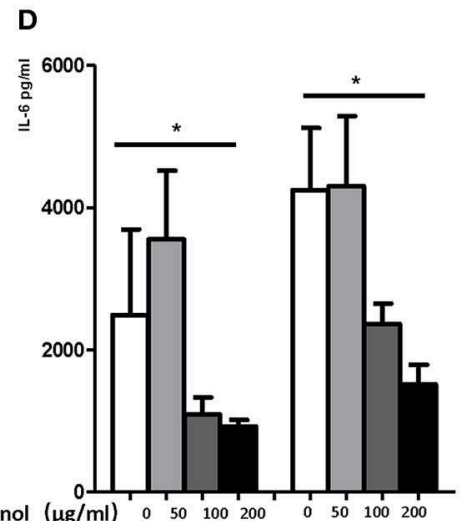

E

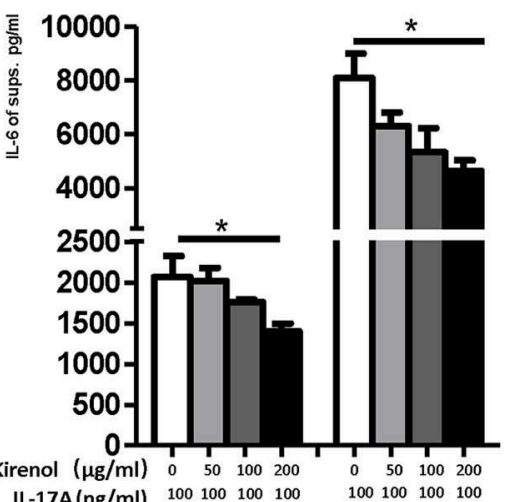

IL-17A (ng/ml)

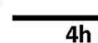

$24 \mathrm{~h}$
$\mathbf{F}$

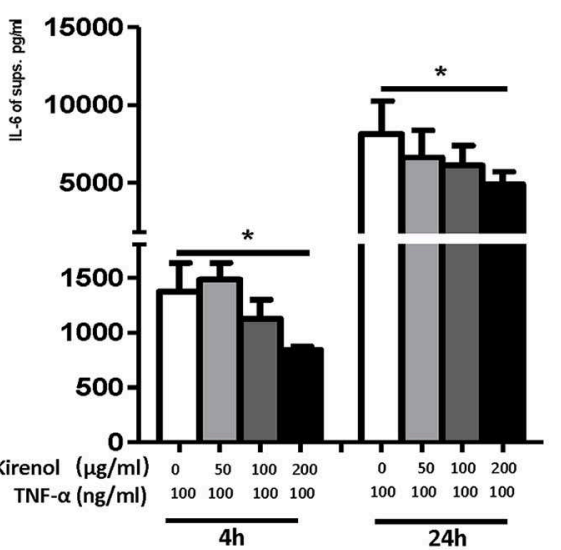

ns

G

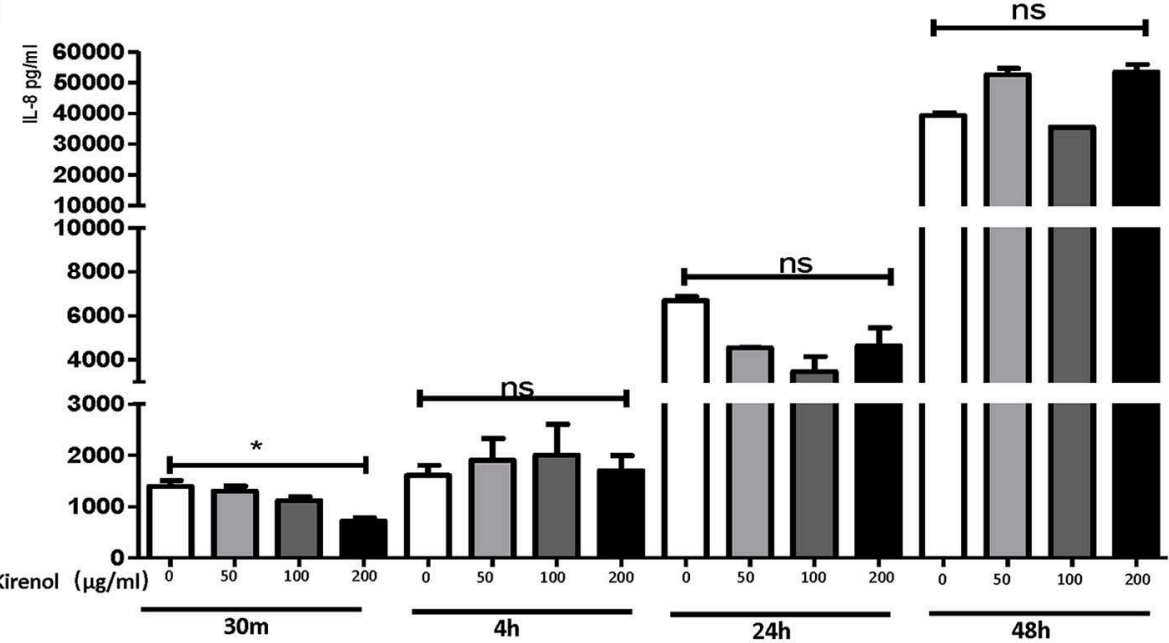

Oug/ml

$50 u g / m l$

ए100ug/ml

$200 u \mathrm{~g} / \mathrm{ml}$

FIGURE 1 | (A) Kirenol inhibited FLS in a dose-dependent manner; (B) Kirenol inhibited the proliferation of FLS stimulated with IL-17A (100 ng/ml); (C) Kirenol inhibited the proliferation of FLS stimulated with TNF- $\alpha$ (100 ng/ml); (D) Kirenol inhibited the secretion of IL-6 by FLS in a dose-dependent manner after 4 and $24 \mathrm{~h}$. (E) Kirenol inhibited the secretion of IL-6 by FLS stimulated with IL-17A in a dose-dependent after 4 and $24 \mathrm{~h}$. (F) Kirenol inhibited the secretion of IL-6 by FLS stimulated with TNF- $\alpha$ in a dose-dependent after 4 and $24 \mathrm{~h}$. (G) Kirenol inhibited the secretion of IL-8 by FLS in a dose-dependent manner after 30 min but not at other time points. ${ }^{*} p<0.05,{ }^{* *} p<0.01$ as assessed by the Kruskal-Wallis test and the Mann-Whitney U-test. 


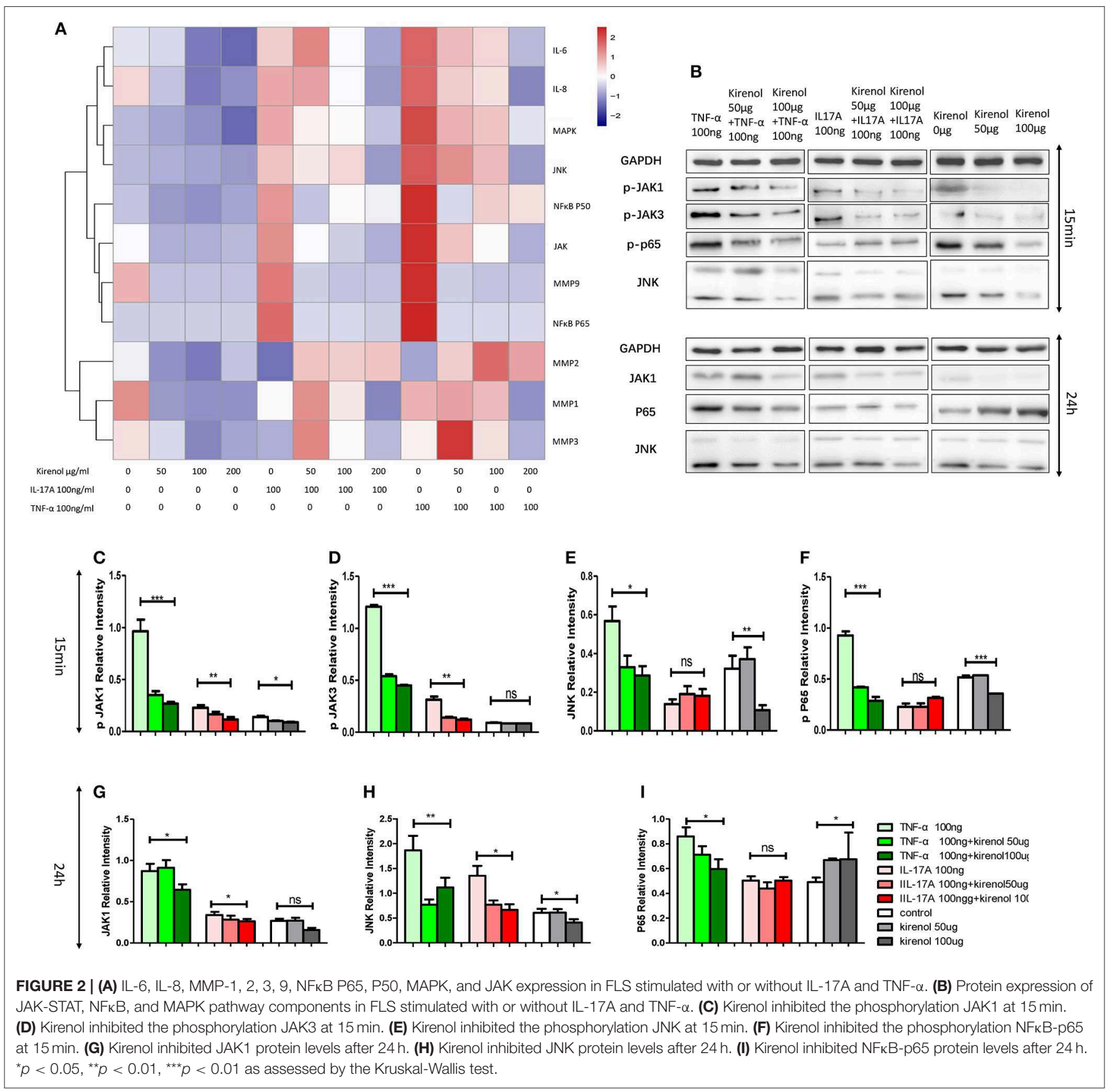

by Kirenol, particularly following TNF- $\alpha$ stimulation. Kirenol had no apparent effect on MMP-1, 2, or 3 expression.

\section{Kirenol Down-Regulates JAK-STAT and NFKB but not MAPK Protein Levels in RA-FLS}

To verify our RT-PCR results, Western blotting was next used to assess levels of key proteins in the MAPK, JAK-STAT, and NFкB pathways in these RA-FLS cells. We found that Kirenol affected the phosphorylation of JAK1 and JAK3 in the JAKSTAT pathways, as well as the phosphorylation of NFKB p65 in the first 15 min following TNF- $\alpha$ stimulation, after which no clear differences in protein phosphorylation were evident (Figures 2B-I).

\section{Kirenol Alters the Migration and Invasion of FLS}

We next assessed the effects of Kirenol on cellular migration and invasion, and found that it inhibited both activities even when cells were stimulated with IL-17 and TNF- $\alpha$. In a wound healing assay, we found that the migratory ability of cells in the Kirenol-treated group was decreased compared with the control group (Figures 3A-D). Consistent with this, significantly fewer 


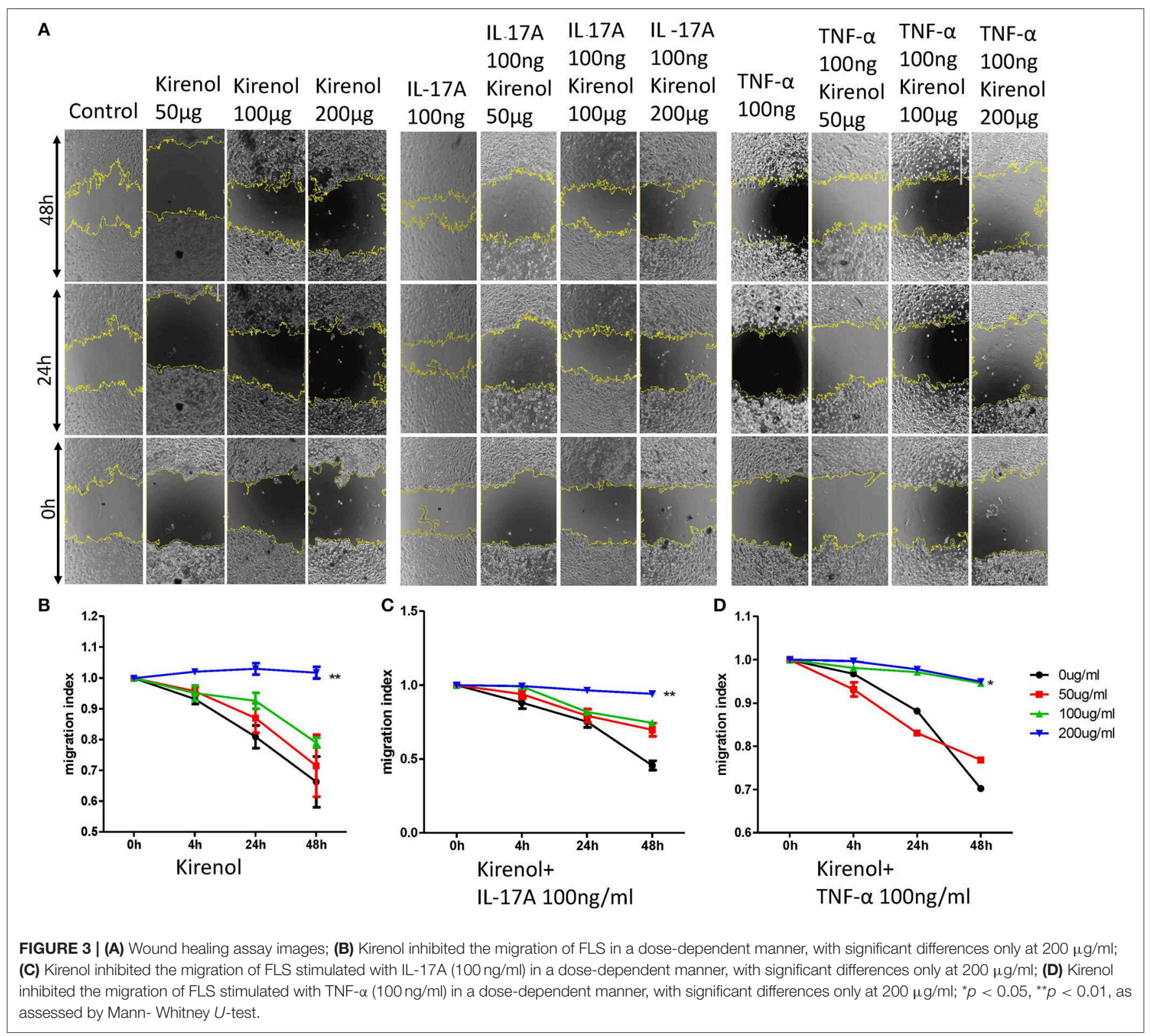

migrated cells were detected upon Kirenol treatment for the invasion assay (Figures $\mathbf{4 A - H}$ ).

\section{Kirenol Alters Arthritic Progression in vivo}

We next sought to extend our findings in vivo, in order to assess whether Kirenol was able to inhibit inflammation in a CIA mouse model of arthritis. We found that a low dose Kirenol $(7.5 \mathrm{mg} / \mathrm{kg})$ was able to delay the onset of arthritis, while a high dose $(30 \mathrm{mg} / \mathrm{kg})$ was able to reduce the incidence of arthritis (Figures 5A,B). Animals in the high dose group also exhibited reduced histological scores (Figures 5D-G), while body weight did not vary significantly at any tested dose (Figure 5C).

When murine serum was assessed via ELISA, we found that Kirenol was able to inhibit the production of TNF- $\alpha$, IL- $1 \beta$, IL6 , and IL-8 of in the serum (Figures 6A-D). Western blotting further confirmed that Kirenol was able to reduce levels of IL6 , IL-8, and TNF- $\alpha$ in the synovium, but only at the higher dose of $30 \mathrm{mg} / \mathrm{kg}$ (Figure 6F). Immunohistochemistry also confirmed that Kirenol can inhibit the levels of IL-6, IL-8, and TNF- $\alpha$ in the synovium, with differences only being significant at the $30 \mathrm{mg} / \mathrm{kg}$ dose (Figures 6E,G).

\section{DISCUSSION}

RA is characterized by the proliferation of synoviocytes in inflamed synovia, and by synoviocyte expression of inflammatory cytokines (15). FLS from RA patients exhibit extended hyperplasia, activation, and other aggressive behaviors such as abnormal migration and invasion $(16,17)$. As such, treatment strategies often focus on controlling the proliferation and 

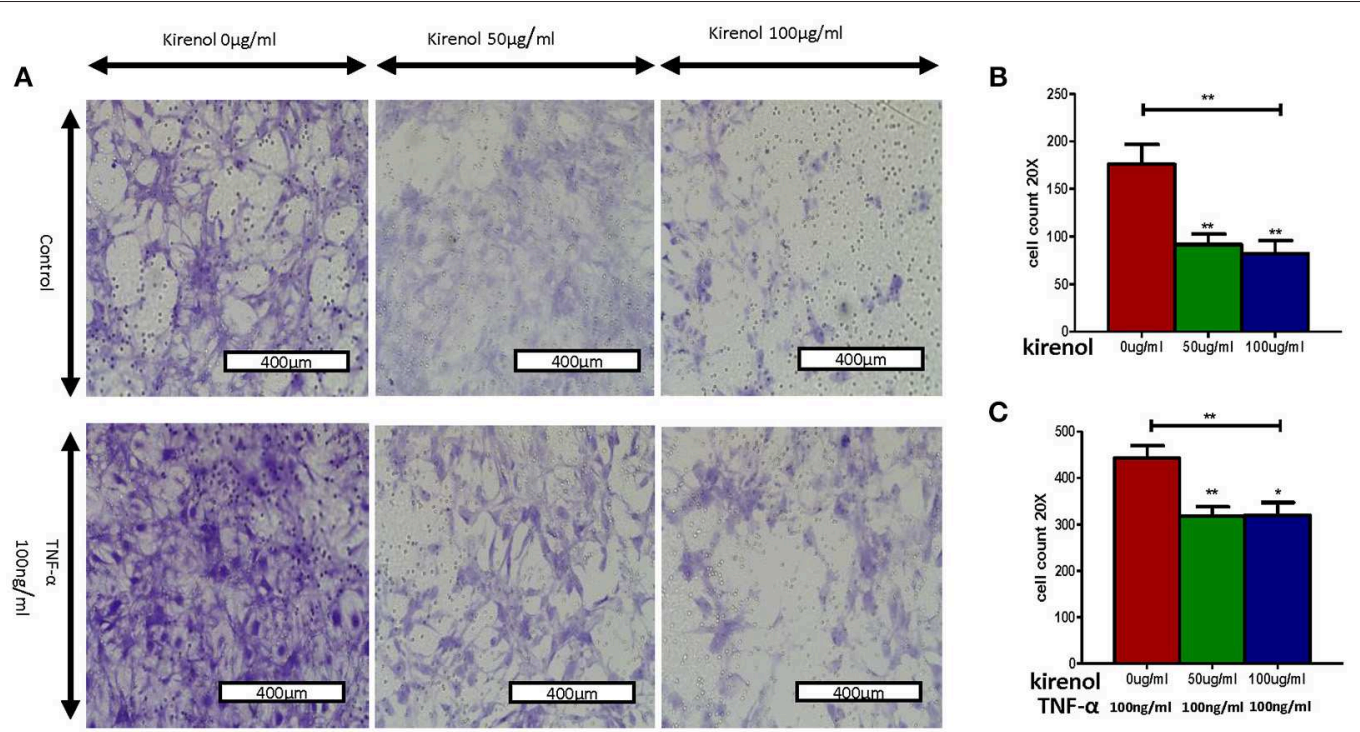

C
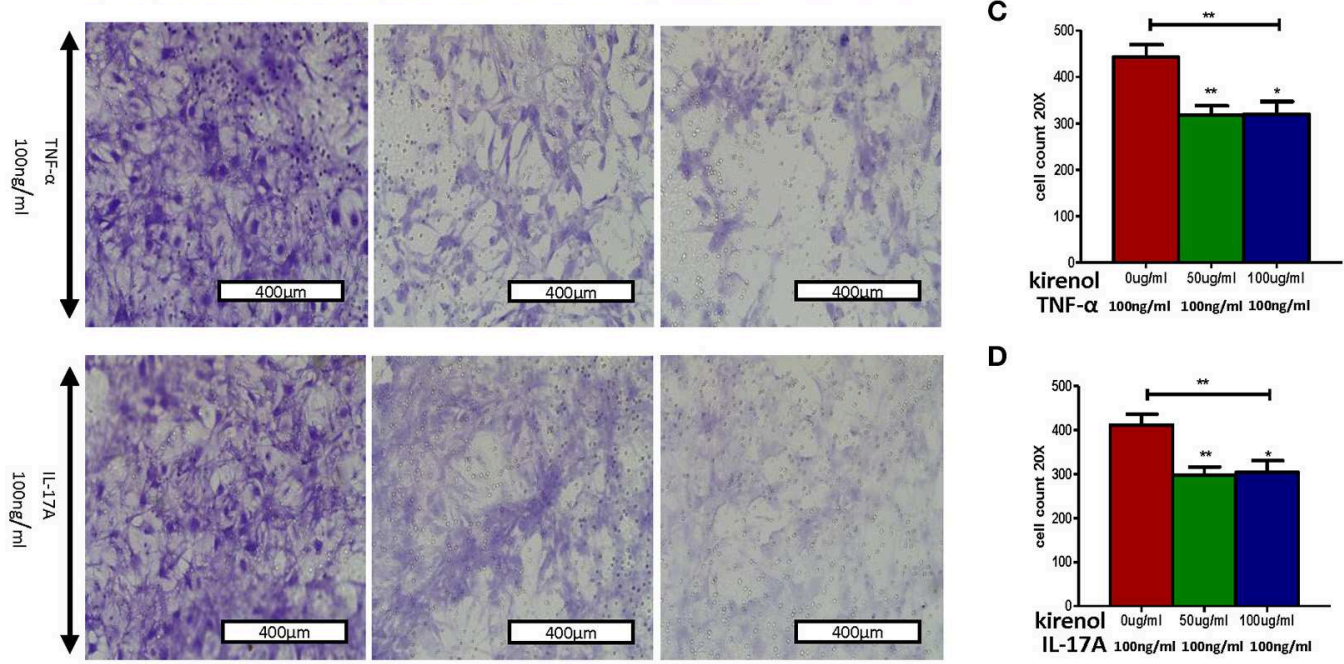

D
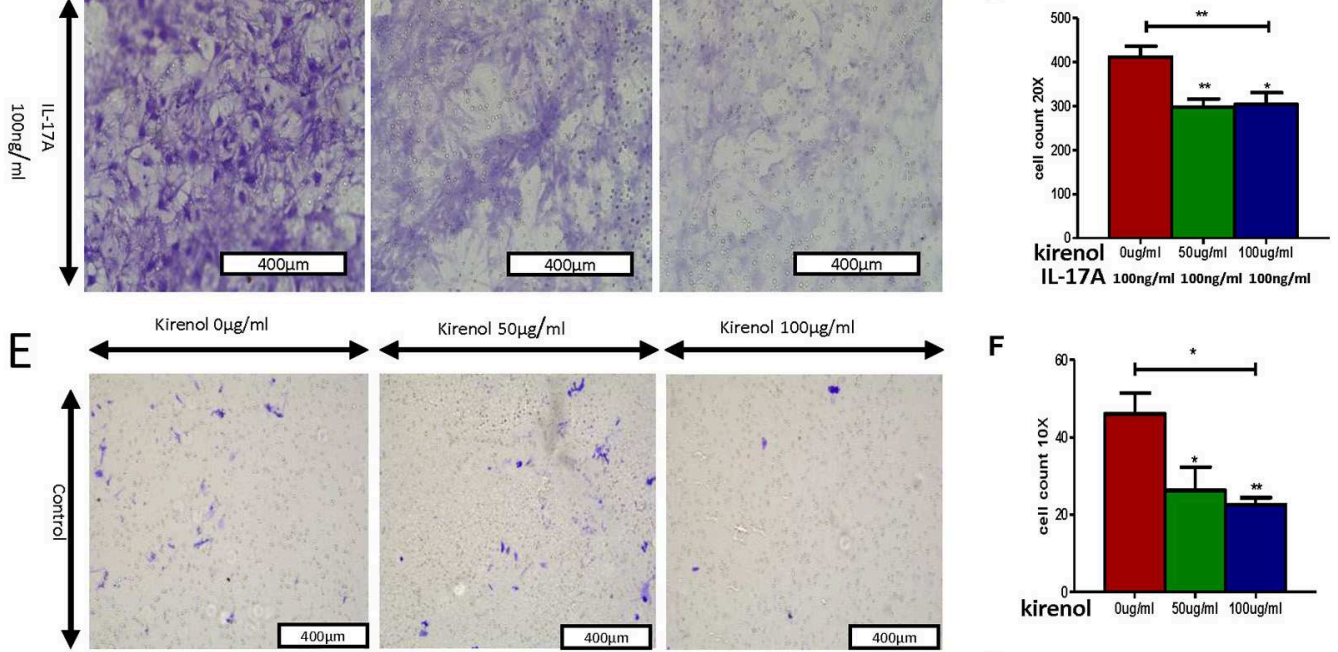

Kirenol $100 \mu \mathrm{g} / \mathrm{ml}$
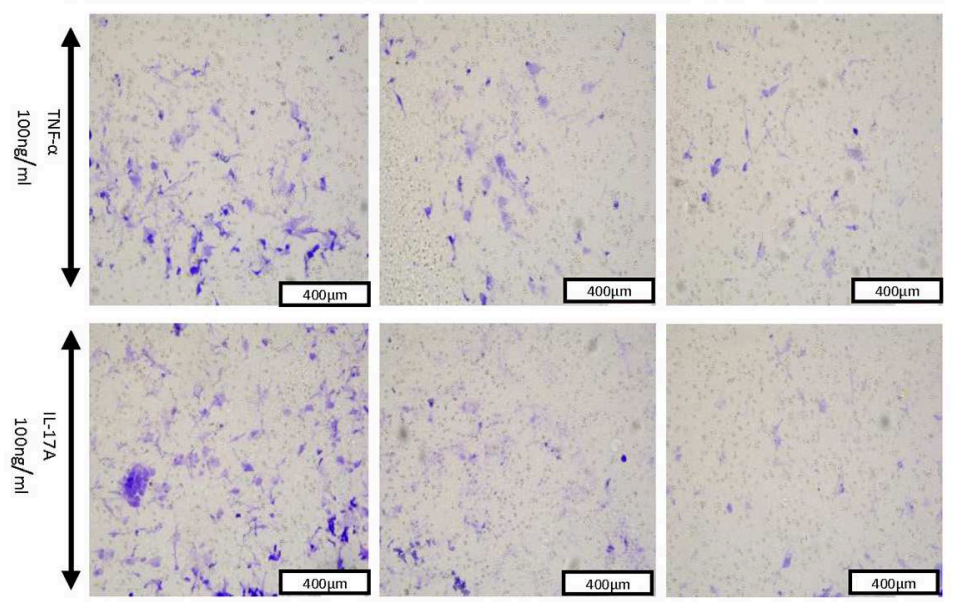

F

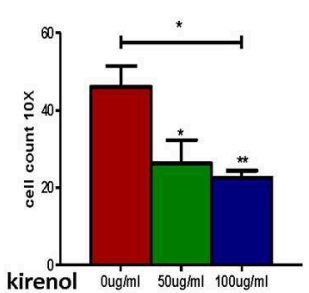

G

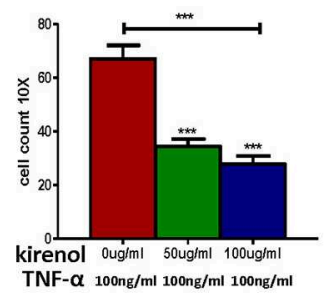

H

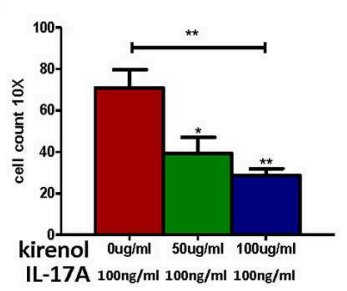

FIGURE 4 | (A) Migration assay images; (B) Kirenol inhibited the migration of FLS; (C) Kirenol inhibited the migration of FLS stimulated with TNF- $\alpha$ (100 ng/ml); (D) Kirenol inhibited the migration of FLS stimulated with IL-17A (100 ng/ml); (E) Invasion assay images; (F) Kirenol inhibited the invasion of FLS; (G) Kirenol inhibited the invasion of FLS stimulated with TNF- $\alpha(100 \mathrm{ng} / \mathrm{ml}) ; \mathbf{( H )}$ Kirenol inhibited the invasion of FLS stimulated with IL-17A $(100 \mathrm{ng} / \mathrm{ml}) ;{ }^{*} p<0.05$, ${ }^{* \star} p<0.01$, ${ }^{\star \star *} p<0.01$ as assessed by the Kruskal-Wallis test and the Mann-Whitney U-test. 


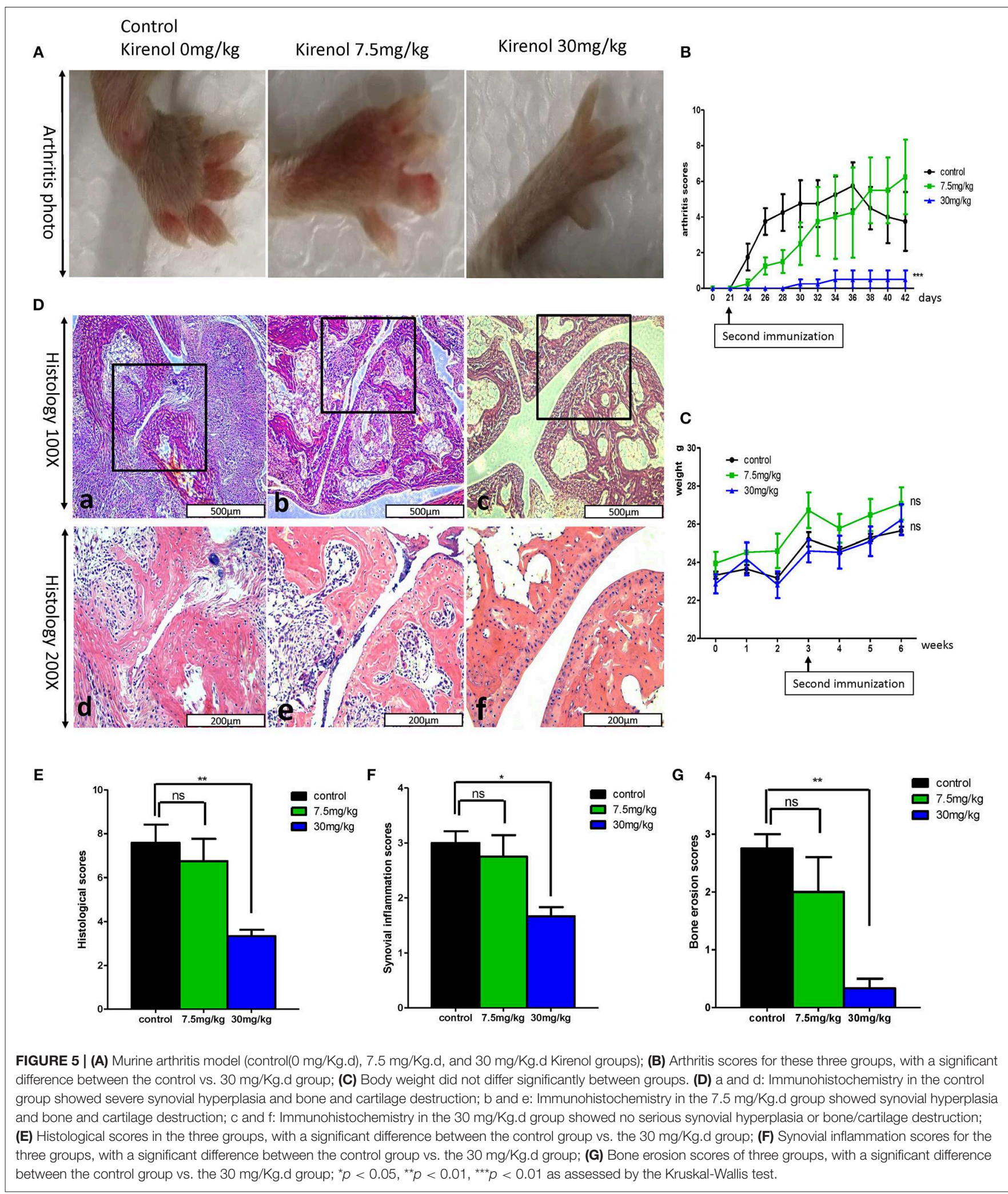

inflammatory nature of these cells. Kirenol is a diterpenoid from Herba Siegesbeckia that has been used to treat RA for centuries. Kirenol has been suggested to exhibit anti-inflammatory and anti-rheumatic activities. Studies have found that Kirenol is effective in rat models of arthritis, but little is known about whether it can directly affect synovial cells. Therefore, in this 


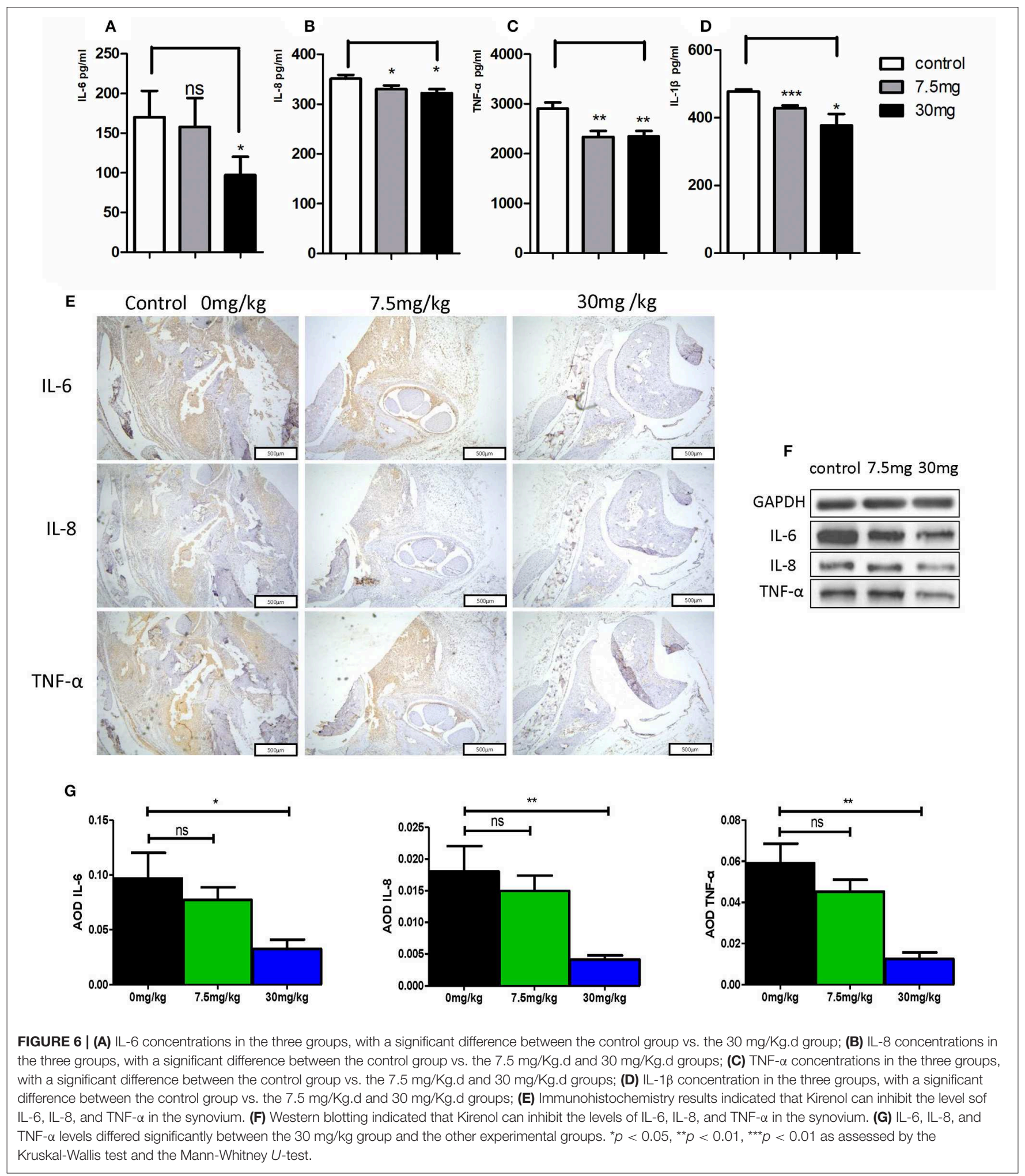

study we designed a series of experiments to observe the effects of Kirenol on FLS both in vitro and in vivo.

In vitro, we found that Kirenol inhibited the proliferation and function of FLS in a dose-dependent manner. Interestingly, Kirenol exerted a more significant anti-proliferative and anti-inflammatory effect when these RA-FLS were first stimulated using TNF- $\alpha$ and IL-17. This confirms our previous findings indicating that Kirenol can dock the TNF- $\alpha$ (6). Furthermore, we found that only high-dose Kirenol (100-200 $\mu \mathrm{g} / \mathrm{ml})$ was able to affect FLS IL- 6 secretion, even following TNF- $\alpha$ and 
IL-17A stimulation. By RT-PCR we further found that Kirenol can inhibit the expression of IL- 6 at the mRNA level, indicating that high-dose Kirenol can readily alter IL- 6 production by FLS. We were only able to detect inhibited IL- 8 production at 30 min after Kirenol treatment in FLS, and such inhibition was absent in cells first treated with cytokines, although at the mRNA level this inhibition was evident. Even so, as these results are inconsistent, it is unclear whether Kirenol can substantially alter IL-8 production in vitro, particularly not in the context of a strong cytokine stimulus. We were not able to detect TNF- $\alpha$ or IL-1 $\beta$ ain FLS supernatants. We further found that RT-PCR that MMP9 can be inhibited by Kirenol in vitro, whereas MMP-1,2, and 3 were not. We did not assess MMP protein levels in this study, and as such this is an important area of future research. We suspect that these proteins may be regulated by Kirenol, given previous work showing that this compound can inhibit MMP-2, 3, 9, and 13 expression in Hs68 human dermal fibroblasts (18).

To evaluate how IL- 6 secretion and FLS function were affected by Kirenol, we next assessed the MAPK, JAK-STAT, and NFкB pathways in FLS cells, revealing that at early time points this compound inhibited the activation of JAK-STAT and NFкB but not MAPK signaling. Many studies have shown that TNF- $\alpha$ and IL-17A signaling through the NFKB pathway regulate the function of FLS in RA (19-21). We found that Kirenol inhibits the function of FLS in response to TNF- $\alpha$ and IL-17A, and so we hypothesized that Kirenol plays a negative role in controlling the activation of the NFאB pathway by blocking TNF- $\alpha$ and IL-17A signaling. Western blotting results were consistent with this hypothesis. Other researchers have also found that TNF- $\alpha$ signaling through JAK-STAT pathways can affect FLS responses (22), suggesting that Kirenol can inhibit responses to TNF signaling via multiple pathways, with similar inhibitory activities also likely in response to IL-6.

Some studies have found that RA-FLS migrate and invade cartilage and bone, leading to vascularization and tissue damage during RA progression (23). We found that Kirenol also inhibited the migration and invasion of FLS in a dose-dependent manner, even in response to TNF- $\alpha$ and IL-17A stimulation, which is significant as both cytokines can strongly promote cellular migration and invasion (24-27). We therefore believe Kirenol has a clear inhibitory effect on the migration and invasion of synoviocytes.

According to previous reports, Kirenol reduces the expression of cytokines in synovial and synovial fluid in a CIA rat model $(28,29)$. Histological evaluation in this study similarly revealed that Kirenol treatment effectively reduced joint inflammation, cartilage damage, and bone erosion, confirming that it helped to protect CIA mice. Our results are similar to those of other studies $(5,30)$. Moreover, we also found that Kirenol

\section{REFERENCES}

1. Aletaha D, Smolen JS. Diagnosis and management of rheumatoid arthritis: a review. JAMA. (2018) 320:1360-72. doi: 10.1001/jama.2018.13103

2. Ospelt C. Synovial fibroblasts in 2017. RMD Open. (2017) 3:e000471. doi: 10.1136/rmdopen-2017-000471 only achieved a protective effect at a dose of $30 \mathrm{mg} / \mathrm{kg}$ in this CIA mice model, with the lower dose only delaying the occurrence of arthritis and ultimately not affecting the disease outcome. No clear cytotoxicity or death was observed at any tested dose, indicating these treatment doses are safe. We further found that Kirenol significantly reduced the levels of TNF- $\alpha$, IL- $1 \beta$, and IL- 6 in murine serum, confirming that this compound exhibits a therapeutic effect in CIA model mice.

Our in vitro and in vivo experiments have thus demonstrated that Kirenol has an excellent ability to inhibit synoviocyte functionality, suggesting that Kirenol has potential as a possible anti-rheumatic drug. This study is, however, limited by the fact that inflammation was only examined in the serum, joints, and synovium. Future studies will need to focus more broadly on how Kirenol affects the immune system in vivo.

\section{CONCLUSIONS}

In this study, we found that Kirenol was able to strongly inhibit FLS proliferation, migration, and invasion, and to inhibit the release of pro-inflammatory IL- 6 by FLS, even when these cells were activated with IL-17A and TNF- $\alpha$. Kirenol is able to mediate this inhibitory activity in FLS via regulating various intracellular pathways. In vivo experiments further confirmed that Kirenol can inhibit bone erosion, synovial hyperplasia, and inflammation in the joints of arthritic mice in a dose dependent manner.

\section{ETHICS STATEMENT}

The study is approved by Ethics Committee of ZhuJiang Hospital of Southern Medical University. The research design and methodology of this study were consistent with the requirements of the 2013 Helsinki Declaration. All study participants provided written informed consent.

\section{AUTHOR CONTRIBUTIONS}

JW, QL, LJ, HD, L-GJ, YQ, B-BL, and XZ performed experiments. JW and QL conceived the study and analyzed the results. JW and QY supervised the study and prepared the manuscript. All authors read and approved the final manuscript.

\section{FUNDING}

This study was supported by the National Natural Science Foundation of China (Grant Nos. 81601397 and 81771727). 
Th1 and th17 cells and inducing apoptosis of effector T cells. Sci Rep. (2015) 5:9022. doi: 10.1038/srep09022

5. Lu Y, Xiao J, Wu ZW, Wang ZM, Hu J, Fu HZ, et al. Kirenol exerts a potent anti-arthritic effect in collagen-induced arthritis by modifying the $\mathrm{T}$ cells balance. Phytomedicine. (2012) 19:882-9. doi: 10.1016/j.phymed.2012.04.010

6. Wu J, Qu Y, Deng J, Liang W, Jiang Z, Lai R, et al. Molecular docking studies of Kirenol a traditional Chinese medicinal compound against rheumatoid arthritis cytokine drug targets (TNF- $\alpha$, IL-1 and IL-6). Biomed Res. (2017) 28:1992-95.

7. Guo Q, Wang Y, Xu D, Nossent J, Pavlos NJ, Xu J. Rheumatoid arthritis: pathological mechanisms and modern pharmacologic therapies. Bone Res. (2018) 6:15. doi: 10.1038/s41413-018-0016-9

8. Wu J, Qu Y, Zhang YP, Deng JX, Yu QH. RHAMM induces progression of rheumatoid arthritis by enhancing the functions of fibroblast-like synoviocytes. BMC Musculoskelet Disord. (2018) 19:455. doi: 10.1186/s12891-018-2370-6

9. Szekanecz Z, Kim J, Koch AE. Chemokines and chemokine receptors in rheumatoid arthritis. Semin Immunol. (2003) 15:15-21. doi: 10.1016/S1044-5323(02)00124-0

10. Kuan WP, Tam LS, Wong CK, Ko FW, Li T, Zhu T, et al. CXCL 9 and CXCL 10 as Sensitive markers of disease activity in patients with rheumatoid arthritis. J Rheumatol. (2010) 37:257-64. doi: 10.3899/jrheum. 090769

11. Zhao J, Ouyang Q, Hu Z, Huang Q, Wu J, Wang R, et al. A protocol for the culture and isolation of murine synovial fibroblasts. Biomed Rep. (2016) 5:171-75. doi: 10.3892/br.2016.708

12. Sekine C, Sugihara T, Miyake S, Hirai H, Yoshida M, Miyasaka N, et al. Successful treatment of animal models of rheumatoid arthritis with smallmolecule cyclin-dependent kinase inhibitors. J Immunol. (2008) 180:1954-61. doi: 10.4049 /jimmunol.180.3.1954

13. Schramm C, Kriegsmann J, Protschka M, Huber S, Hansen T, Schmitt E, et al. Susceptibility to collagen-induced arthritis is modulated by TGFbeta responsiveness of T cells. Arthritis Res Ther. (2004) 6:R114-9. doi: $10.1186 / \operatorname{ar} 1039$

14. Miranda JP, Camoes SP, Gaspar MM, Rodrigues JS, Carvalheiro M, Barcia RN, et al. The secretome derived from 3D-cultured umbilical cord tissue MSCs counteracts manifestations typifying rheumatoid arthritis. Front Immunol. (2019) 10:18. doi: 10.3389/fimmu.2019.00018

15. McInnes IB, Schett G. Pathogenetic insights from the treatment of rheumatoid arthritis. Lancet. (2017) 389:2328-37. doi: 10.1016/S0140-6736(17) 31472-1

16. Bartok B, Firestein GS. Fibroblast-like synoviocytes: key effector cells in rheumatoid arthritis. Immunol Rev. (2010) 233:233-55. doi: 10.1111/j.0105-2896.2009.00859.x

17. Xu S, Xiao Y, Zeng S, Zou Y, Qiu Q, Huang M, et al. Piperlongumine inhibits the proliferation, migration and invasion of fibroblast-like synoviocytes from patients with rheumatoid arthritis. Inflamm Res. (2018) 67:233-43. doi: $10.1007 / \mathrm{s} 00011-017-1112-9$

18. Kim J, Kim MB, Yun JG, Hwang JK. Protective effects of standardized siegesbeckia glabrescens extract and its active compound kirenol against UVB-induced photoaging through inhibition of MAPK/NFkappaB pathways. J Microbiol Biotechnol. (2017) 27:242-50. doi: $10.4014 /$ jmb.1610.10050

19. Hwang SY, Kim JY, Kim KW, Park MK, Moon Y, Kim WU, et al. IL-17 induces production of IL-6 and IL-8 in rheumatoid arthritis synovial fibroblasts via
NF-kappaB- and PI3-kinase/Akt-dependent pathways. Arthritis Res Ther. (2004) 6:R120-8. doi: 10.1186/ar1038

20. Makarov SS. NF-kappa B in rheumatoid arthritis: a pivotal regulator of inflammation, hyperplasia, and tissue destruction. Arthritis Res. (2001) 3:2006. doi: $10.1186 /$ ar300

21. Lee A, Qiao Y, Grigoriev G, Chen J, Park-Min KH, Park SH, et al. Tumor necrosis factor alpha induces sustained signaling and a prolonged and unremitting inflammatory response in rheumatoid arthritis synovial fibroblasts. Arthritis Rheum. (2013) 65:928-38. doi: 10.1002/art.37853

22. Malemud CJ. Negative regulators of JAK/STAT signaling in rheumatoid arthritis and osteoarthritis. Int J Mol Sci. (2017) 18:E484. doi: 10.3390/ijms18030484

23. Huber LC, Distler O, Tarner I, Gay RE, Gay S, Pap T. Synovial fibroblasts: key players in rheumatoid arthritis. Rheumatology. (2006) 45:669-75. doi: 10.1093/rheumatology/kel065

24. Li G, Zhang $\mathrm{Y}$, Qian $\mathrm{Y}$, Zhang $\mathrm{H}$, Guo $\mathrm{S}$, Sunagawa $\mathrm{M}$, et al. Interleukin-17A promotes rheumatoid arthritis synoviocytes migration and invasion under hypoxia by increasing MMP2 and MMP9 expression through NF-kappaB/HIF-1alpha pathway. Mol Immunol. (2013) 53:227-36. doi: 10.1016/j.molimm.2012.08.018

25. Pickens SR, Volin MV, Mandelin AM II, Kolls JK, Pope RM, Shahrara S. IL-17 contributes to angiogenesis in rheumatoid arthritis. J Immunol. (2010) 184:3233-41. doi: 10.4049/jimmunol.0903271

26. Hot A, Miossec P. Effects of interleukin (IL)-17A and IL-17F in human rheumatoid arthritis synoviocytes. Ann Rheum Dis. (2011) 70:727-32. doi: $10.1136 /$ ard.2010.143768

27. Zeng S, Wang K, Huang M, Qiu Q, Xiao Y, Shi M, et al. Halofuginone inhibits TNF-alpha-induced the migration and proliferation of fibroblastlike synoviocytes from rheumatoid arthritis patients. Int Immunopharmacol. (2017) 43:187-94. doi: 10.1016/j.intimp.2016.12.016

28. Huo L, Jiang Z, Lei M, Wang X, Guo X. Simultaneous quantification of Kirenol and ent-16beta,17-dihydroxy-kauran-19-oic acid from Herba Siegesbeckiae in rat plasma by liquid chromatography-tandem mass spectrometry and its application to pharmacokinetic studies. J Chromatogr B Analyt Technol Biomed Life Sci. (2013) 937:18-24. doi: 10.1016/j.jchromb.2013. 08.019

29. Kim MB, Song Y, Kim C, Hwang JK. Kirenol inhibits adipogenesis through activation of the Wnt/beta-catenin signaling pathway in 3T3-L1 adipocytes. Biochem Biophys Res Commun. (2014) 445:433-8. doi: 10.1016/j.bbrc.2014.02.017

30. Lu Y, Xiao J, Wu Z, Wang Z, Fu H, Chen Y, et al. Effects of Kirenol on bovine type II collagen-induced rat lymphocytes in vivo and in vitro. Nan Fang Yi Ke Da Хие Хие Bao. (2012) 32:1-6.

Conflict of Interest Statement: The authors declare that the research was conducted in the absence of any commercial or financial relationships that could be construed as a potential conflict of interest.

Copyright () $2019 \mathrm{Wu}$, Li, Jin, Qu, Liang, Zhu, Du, Jie and Yu. This is an open-access article distributed under the terms of the Creative Commons Attribution License (CC $B Y)$. The use, distribution or reproduction in other forums is permitted, provided the original author(s) and the copyright owner(s) are credited and that the original publication in this journal is cited, in accordance with accepted academic practice. No use, distribution or reproduction is permitted which does not comply with these terms. 